\title{
Psychotropic medications and cutaneous adverse drug reactions
}

\author{
Otheman $\mathrm{Y}^{1 *}$, El benaye $\mathrm{J}^{2}$, kadiri $\mathrm{M}^{1}$, Mehssani $\mathrm{J}^{1}$ and Bichra $\mathrm{MZ}^{1}$ \\ ${ }^{1}$ Department of Psychiatry, Mohammed-V Military Teaching Hospital. Faculty of medicine and pharmacy, Mohammed V University, Souissi, Rabat, Morocco \\ ${ }^{2}$ Department of Dermatology, Military Hospital Moulay Ismail, Meknès. Faculty of medicine and pharmacy, Sidi Mohamed Ben Abdellah University, Fes, Morocco
}

\section{Introduction}

Cutaneous adverse drug reactions (CADRs) are common and potentially serious side effects. Among psychotropic medications, the most incriminated molecules are anticonvulsants, used as mood stabilizers in affective disorders, such as carbamazepine, lamotrigine and sodium valproate. However, other classes of psychotropic drugs may also be involved, such as antidepressants and anxiolytics. In this article, we will present four illustrated cases of CADRs induced by different types of psychotropic drugs.

\section{Clinical Images}

Case 1: A 24-year-old man with an anxiety disorder, who was treated by an antidepressant of the SSRI class (paroxetine) for a month. He presented an erythematous and vesicular eruption, located strictly on the photo-exposed zones, respecting the nasolabial folds and the submandibular region, evoking a photo-toxicity (Figures 1 and 2).

Case 2: During a manic episode, in a 40 -year-old woman followed for bipolar disorder, the psychiatrist increased the dosage of the mood stabilizing treatment: carbamazepine from 400 to $800 \mathrm{mg} /$ day. Two weeks later, the patient developed oral erosions with red and blackish crusts, and purpuric lesions of limbs, some of which having an aspect of target lesions, typically found in the Stevens Johnson syndrome (Figures 3 and 4).

Case 3: After a period of discontinuation of his treatment, a 46-year-old bipolar patient was given sodium valproate at a dose of $1 \mathrm{gr} / \mathrm{d}$ and presented one week later a diffuse maculopapular rash in purpuric and scaly areas with mucosal involvement, suggestive of CADRs erythroderma type (Figures 5, 6 and 7).

Case 4: After an isolated episode of insomnia, a 32-year-old patient was given benzodiazepines (bromazepam). Four days later, he

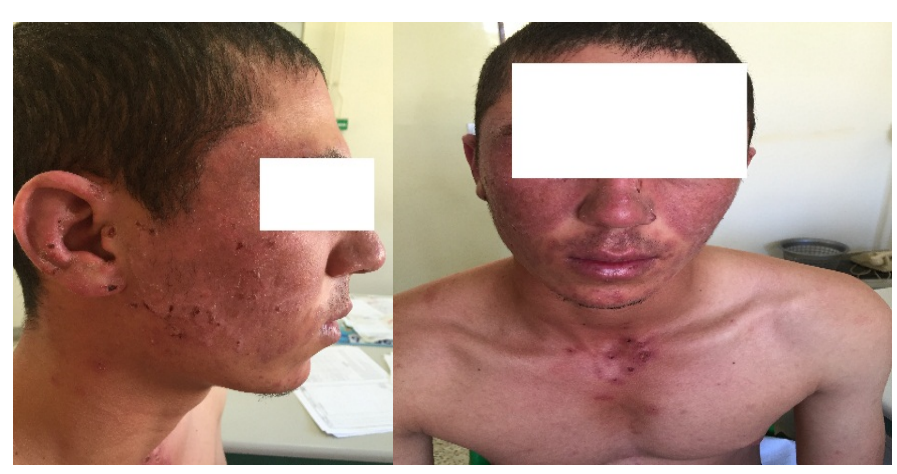

Figures 1 and 2. Photo-toxicity induced by paroxetine

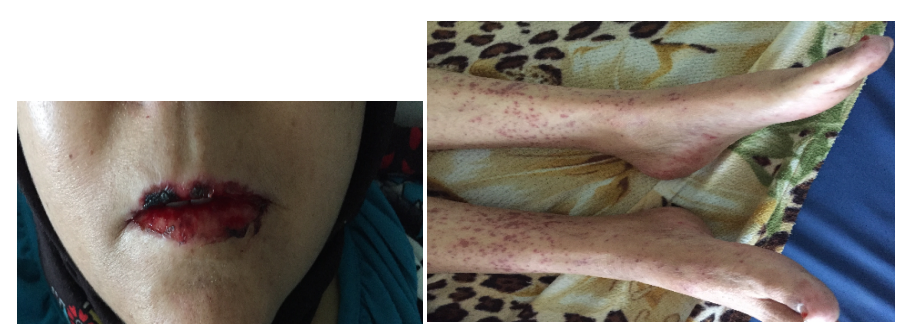

Figures 3 and 4 . Stevens johnson syndrome induced by carbamazepine
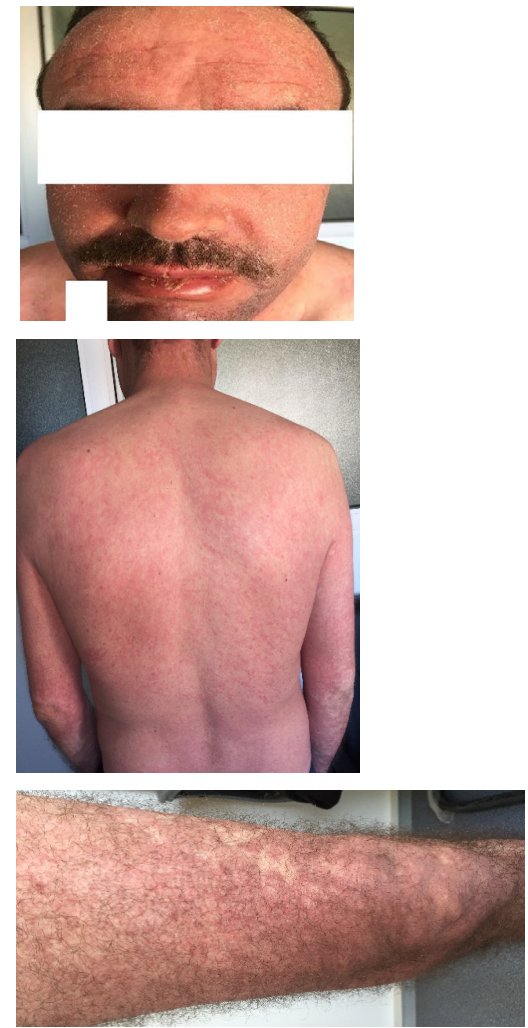

Figures 5,6 and 7. Erythroderma induced by sodium valproate

*Correspondence to: Yassine otheman, Assistant Professor of Psychiatry, Mohamed V Military Teaching Hospital, Rabat, Morocco, E-mail: yothman12@yahoo.fr

Received: June 06, 2018; Accepted: June 27, 2018; Published: July 02, 2018 
presented confluent cutaneous and mucosal erosions of the external genitals, evolving after one week to a hyperpigmentation, evoking a fixed pigmented erythema (Figures 8 and 9).

\section{Discussion}

CADRs are common, and usually have minimal associated morbidity and mortality risk. However, there are reactions such as Stevens Johnson syndrome (SJS), toxic epidermal necrolysis (TEN), and drug reaction with eosinophilia and systemic symptoms (DRESS) which are associated with significant morbidity and mortality $[1,2]$. The diagnosis is based on the clinical presentation, the chronology of the appearance of the lesions after taking the suspected drug, and the elimination of differential diagnoses [3].

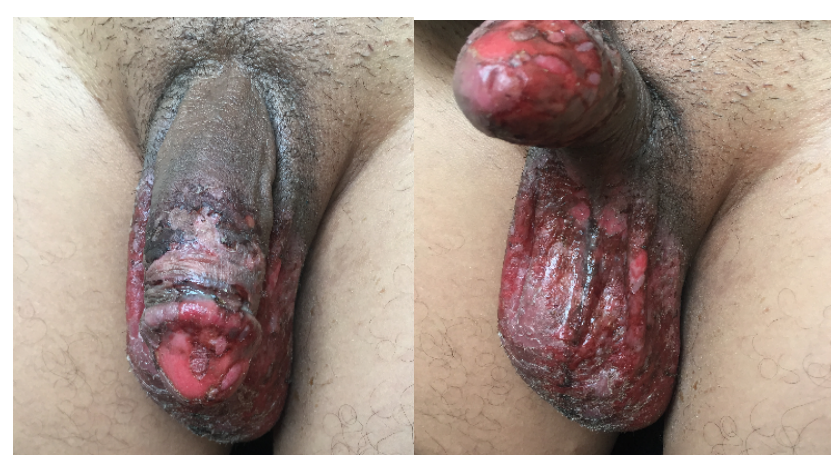

Figures 8 and 9. Fixed pigmented erythema induced by benzodiazepines

\section{Conclusion}

The prevalence of CADRs is variable around the world; this may be due to genetic variability, while immunological factors are mainly mentioned as predisposing to these cutaneous side effects [4,5].

Any rash or unusual dermatological sign must attract attention and motivate urgent consultation with a specialist. It is important that the clinician recognize signs and symptoms of severe CADRs. Stopping treatment as soon as possible is advised. The incriminated molecules should be reported in pharmacovigilance, and the patient must be informed of the risk of further intake.

\section{Conflicts of Interest}

Authors declare no conflict of interest.

\section{References}

1. Swanson L, Colven RM (2015) Approach to the Patient with a Suspected Cutaneous Adverse Drug Reaction. Med Clin North Am 99:1337-1348.

2. Naldi L, Crotti S (2014) Epidemiology of cutaneous drug-induced reactions. G Ital Dermatol Venereol 149: 207-218.

3. Vignes LB, Allanore VL (2015) La Revue de médecine interne 36: 256-270.

4. Sukasem C, Puangpetch A, Medhasi S, Tassaneeyakul W (2014) Pharmacogenomics of drug-induced hypersensitivity reactions: challenges, opportunities and clinical implementation. Asian Pac J Allergy Immunol 32: 111-23.

5. Chung WH, Wang CW, Dao RL (2016) Severe cutaneous adverse drug reactions. $J$ Dermatol 43: 758-766.

Copyright: (C2018 Otheman Y. This is an open-access article distributed under the terms of the Creative Commons Attribution License, which permits unrestricted use, distribution, and reproduction in any medium, provided the original author and source are credited. 